\title{
Comment
}

Neuro epidemiology

\section{On the Epidemiology of Multiple Sclerosis in the Middle East and North Africa}

\author{
John F. Kurtzke \\ Georgetown University School of Medicine, Washington, D.C., USA
}

Professor Sahraian and his colleagues from Tehran, Iran, have presented a unique summation of the developing evolution of the epidemiology of multiple sclerosis (MS) in a region of growing importance, and a region about which most of us have little awareness [1] (Neuroepidemiology in press). The trend of the prevalence of MS with a high frequency in the north and with a low frequency in the south has long disappeared from Europe and has been reducing in the Americas as well. Citing recent scattered reports suggesting that regions of the Middle East and North Africa, situated largely between the 15th and 25th parallels of latitude, may also be joining in this trend of increasing MS, they have analyzed all relevant publications that appeared between January 1, 1985 and August 1,2014; 52 such reports were included in their analysis.

Results were presented in several dense tables that I found difficult to absorb, including one (table 3) titled 'meta-analysis of the pooled estimates for prevalence, mean age at disease onset, and female proportion of patients'. The first of these cited a pooled estimate of prevalence of 51.52 per 100,000 population (LL 36.10, UL $66.95), 4,835.85$ heterogeneity statistic, and I-squared of 99.8\%; this last figure, which with these other measures I had to look up, I think means that almost all the variance in the meta-analysis was attributable to study heterogeneity. 'A moment-based random model was used for esti- mating pooled measures because of heterogeneity of studies'. This poor old clinician had difficulty interpreting such pooled measures and believed it was far preferable simply to look at the data.

Included here is a table 1, which I constructed (from their tables 1 and 2) that summarizes to me their major epidemiologic findings. Prevalence day was presumably in the last of the cited study years. Half the surveys were, understandably perhaps, from Iran.

In that country, Sherlock Holmes' dog that did nothing in the nighttime is relevant: two of its three largest cities were cited: Tehran and Isfahan/Esfahan; the third city, Mashhad, was not cited presumably because there was little MS found there. Mashhad is in the far northeastern corner of the country, near Turkmenistan. Please see the map (fig. 1).

Six of the surveys for Iran were from Isfahan with the earliest conducted in 2004-2005 showing the lowest prevalence rate of 36 per 100,000; this increased thereafter to 86 and 73 in 2003-2013 and 2003-2010, respectively. Incidence rates of 9 per 100,000 in these two Isfahan studies suggest an even higher prevalence in the future. Rates in Tehran clearly increased markedly over time: from the authors' Complementary table 4 , the change was from 60 cases in 8.8 million population in 1989 to 763 in 13.4 million in 2006. Much of the marked population growth in Tehran was attributed to immigrants, presumably those
John F. Kurtzke, MD, FACP, FAAN

E-Mail kurtzke2@aol.com 
Table 1. MS in Middle East and North Africa: point prevalence and average annual incidence rates per 100,000 population, from tables 1,2 of Heydarpour et al. [1]

\begin{tabular}{|c|c|c|c|c|}
\hline Country & Area & Years & Prevalence & Incidence \\
\hline Egypt & Red sea govt & 2009-2012 & 14 & - \\
\hline \multirow[t]{17}{*}{ Iran } & \multirow[t]{6}{*}{ Isfahan } & 2003-2013 & 86 & 9 \\
\hline & & $2003-2010$ & 73 & 9 \\
\hline & & $2003-2006$ & 44 & 4 \\
\hline & & 2004-2005 & 36 & - \\
\hline & & 1989-2009 & 51 & 3 \\
\hline & & 1999-2008 & 52 & - \\
\hline & Tehran & $1991-2011$ & 74 & - \\
\hline & N. Khorasan Pr & $2005-2011$ & 13 & - \\
\hline & northeast & 2009 & 9 & - \\
\hline & Kerman $\mathrm{Pr}$ & report 2013 & 32 & - \\
\hline & Kermanshah & 2012 & 43 & - \\
\hline & southwest & $2005-2011$ & 25 & - \\
\hline & - & 1997-2009 & 16 & 2 \\
\hline & Qom & 2011 & 50 & - \\
\hline & E. Azerbaijan Pr & 2005-2009 & 28 & - \\
\hline & Mazandran Pr & 2007 & 20 & - \\
\hline & - & 2011 & 45 & - \\
\hline \multirow[t]{3}{*}{ Jordan } & - & 2004-2005 & 38 & 1 \\
\hline & - & 1992-1993 & 32 & - \\
\hline & - & 1992-1993 & 20 & - \\
\hline \multirow[t]{3}{*}{ Kuwait } & - & 2010-2013 & 85 & 7 \\
\hline & - & 1988 & 10 & - \\
\hline & - & 1991-2000 & 15 & 2 \\
\hline Libya & - & 1982-1984 & 4 & 1 \\
\hline \multirow[t]{4}{*}{ Turkey } & N. Caucasian & 2008-2009 & 69 & - \\
\hline & Multiple & 2006 & 51 & - \\
\hline & 3 rural Black sea & $2002-2003$ & 101 & - \\
\hline & Edine city & 2003-2004 & 34 & - \\
\hline \multirow[t]{2}{*}{ Oman } & - & 1990-2000 & 4 & - \\
\hline & - & 1992-1996 & 1 & - \\
\hline Qatar & - & 2010 & 65 & - \\
\hline Saudi Arabia & - & 1986-1995 & $25^{*}$ & - \\
\hline UAEmirate & - & 2000-2007 & 55 & 7 \\
\hline
\end{tabular}

* Rate per 100,000 hospital patients in Riyadh.

displaced by the ongoing wars and unrest in the area for the last quarter century. Both these centers, as well as Mashhad, reflect large catchment areas and not just the cities. Most of the higher prevalence areas of Iran are located in the western half of the country up to its western borders on the Persian Gulf and the east coast of Iraq.

Of the other countries cited, Kuwait had been increasing from a prevalence of 10 in 1988 to 85 in 2013, with an incidence of 7 for 2010-2013. Qatar in 2010 had a prevalence of 65; in United Arab Emirates it was 55 in 2007 with an incidence of 7 in 2000-2007. Oman remained with a low prevalence through 2000 . No proper prevalence rate is provided for Saudi Arabia. Turkey, which may be said to share European rates, is high. Jordan's rate increased up to a prevalence of 38 and an incidence of 1 in 20042005. Egypt in the Sinai Peninsula showed 2012 prevalence of 14, and an old survey in Libya, to the west of Egypt on the Mediterranean Sea, recorded a 1984 prevalence of 4 per 100,000.

The authors commented that 'the increase in disease prevalence in various MENA [Middle East and North Africa] countries might be due to western lifestyle changes in these countries. Smoking, sun protection products, low vitamin D levels and environmental factors such as air pollution might be possible candidates'. But to me their data indicate a very recent marked and concomitant increase in the disease across several of these lands.

I have a rather different explanation to offer, and that lies in my interpretation of the epidemiology of this disease from its earliest developments, into which the Middle East results seem to me to fit as well The basis for the statements to follow may be found in two recent summations, an Occasional Paper titled 'Epidemiology in multiple sclerosis: a pilgrim's progress' [2] and its documentation and elaboration in a Monograph called 'How Far Can Epidemiology Take Us in Finding the Cause of Multiple Sclerosis?' (Monograph 1, 2014, Department of Veterans Affairs MS Center of Excellence - East) [3].

To summarize, early community surveys, all of which were published after World War II, divided the world into regions of high- (30+ per 100,000), medium- (5-29) and low-prevalence $(<5)$ rates. Regions with a high rate then comprised northwestern Europe, southern Canada, northern US, New Zealand, and southeastern Australia. Since then more and more of the world has joined the first two classes. Early nationwide surveys of each country of Fennoscandinavia and of Switzerland showed high rates in single contiguous regions of each land. These suggested an origin of MS in south-central Sweden which spread to its neighbors and, in the 17th century, southward on to the continent. Emigrants from the high regions of Sweden and Norway introduced MS to the states of Wisconsin and Minnesota in the latter 19th century, followed later by diffusion across all the northern states and more recently into the south.

Migration of patients from higher to lower areas indicated acquisition of the disease by about age 15 with prolonged latency before clinical onset. Reverse migrations 


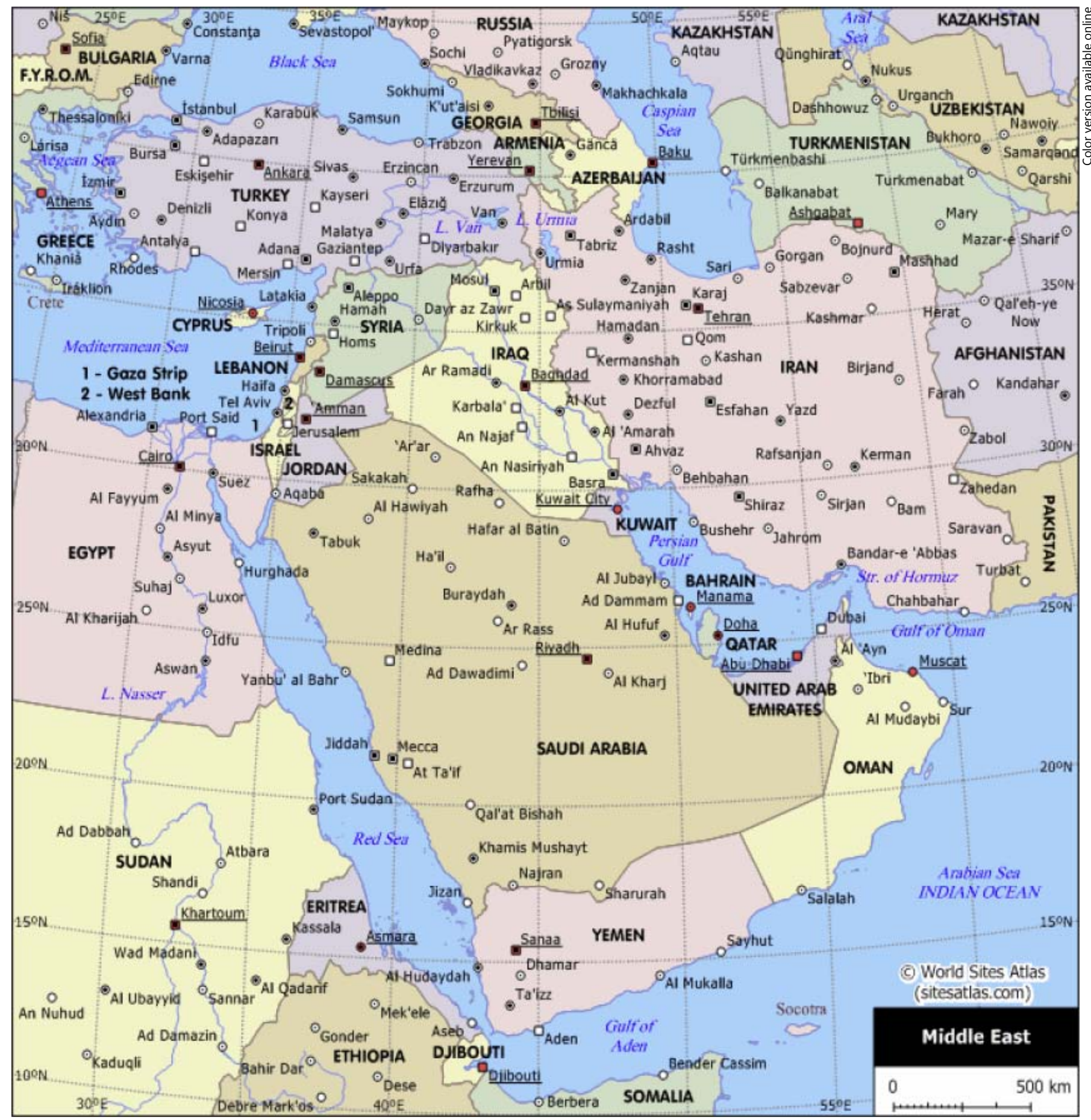

Fig. 1. Geopolitical map of Middle East. (C) World Sites Atlas (sitesatlas.com).

showed susceptibility limited to a period from age 11 to 45. Variations in herd immunity would explain such differences in periods required for acquisition and latency, from 4 and 15-20 years in high areas, to 3 and 10 years for migrants from medium to high, and to 2 and 5 years in virgin populations, where its first appearance or marked increase in some lands occurred as epidemics.

A type 1 epidemic of MS occurred in the Faroe Islands, then part of the Kingdom of Denmark, with the first case in 1943 and peak incidence rates of 10 per 
100,000 in 1945 and 1946. The disease was introduced by British military forces who occupied the islands from April 1940 to September 1945. Similar type 2 epidemics happened in Shetland-Orkney, north of Scotland, and Iceland (and probably northern Jutland in Denmark), all after the occupation by the military in WWII, respectively British, British and American (and German). The best-studied epidemic, that of the Faroes, indicated that the British had brought with them a specific, previously unknown, widespread, asymptomatic, persistent infection that we called the primary multiple sclerosis affection (PMSA). This precursor stage we believe was a newly acquired acute infectious gastroenteritis affect- ing Faroese of all ages, and that among those infected at age 11 to 45 , the PMSA cases, this persisted in the gut up to age 25-30, with early spread to intestinal lymph nodes and, in a few of these, with further (lifelong?) spread into the CNS via blood and cerebrospinal fluid. These cases resulted in the cases of clinical MS whose onset defined this epidemic, which I think is a model for MS elsewhere. A search conducted for such a presumed persistent neurotropic enterovirus in current patients would provide a test of this somewhat involved hypothesis for what I believe is the cause of MS in general, including its recent high prevalence in the Middle East.

\section{References}

1 Heydarpour P, Khoshkish S, Abtahi S, Moradi-Lakeh M, Sahraian MA: Multiple sclerosis epidemiology in Middle East and North Africa: a systematic review and meta-analysis. Neuroepidemiology 2015, in press.

2 Kurtzke JF: Epidemiology in multiple sclerosis: a pilgrim's progress. Brain 2013;136: 2904-2917.
3 Kurtzke JF: How Far Can Epidemiology Take Us in Finding the Cause of Multiple Sclerosis? Baltimore, MD, Department of Veterans Affairs MS Center of Excellence - East: Monograph 1, 2014. http://www.va.gov/MS/ professionals/research/index.asp or from the author atkurtzke2@aol.com. 